\title{
Stand volume models based on stable metrics as from multiple ALS acquisitions in Eucalyptus plantations
}

\author{
Eric Bastos Görgens • Petteri Packalen • André Gracioso Peres da Silva • \\ Clayton Alcarde Alvares • Otavio Camargo Campoe • \\ José Luiz Stape • Luiz Carlos Estraviz Rodriguez
}

Received: 6 July 2014 / Accepted: 5 January 2015 / Published online: 28 January 2015

(C) INRA and Springer-Verlag France 2015

\begin{abstract}
- Key message The selection of stable metrics can generate reliable models between different data sets. The height metrics provide the greatest stability, specifically the higher percentiles and the mode. Height metrics transfer more predictive power than density metrics.

- Context In forestry, there is an increasing development of aerial laser scanning (ALS). The flight missions that permit to record ALS point clouds are not yet standardized. Therefore, there is a need to identify the metrics that permit to infer robust forest stand estimates from the different point cloud acquisitions.
\end{abstract}

Handling Editor: Jean-Michel Leban

Contribution of the co-authors Eric Bastos Görgens Contribution was developing the methodology, running the analysis, and writing the paper. Petteri Packalen was supervising and writing the paper.

André Gracioso Peres da Silva was running the analysis and reviewing the paper.

Clayton Alcarde Alvares was writing and reviewing the paper.

Otavio Camargo Campoe was executing the field data acquisition campaign.

José Luiz Stape was coordinating the EUCFLUX project.

Luiz Carlos Estraviz Rodriguez was supervising and writing the paper.

E. B. Görgens $(\varangle) \cdot$ A. G. P. da Silva • L. C. E. Rodriguez Forest Sciences Department, University of São Paulo-ESALQ,

13416-000 Piracicaba, SP, Brazil

e-mail: gorgens@usp.br

A. G. P. da Silva

e-mail: andregracioso@gmail.com

L. C. E. Rodriguez

e-mail: lcer@usp.b

\section{P. Packalen}

Faculty of Science and Forestry, University of Eastern Finland, P.O. Box 111, FI-80101 Joensuu, Finland

e-mail: petteri.packalen@uef.fi
- Aims The aim of this study is to identify stable metrics derived from different ALS data sets to be used as independent variable in stand volume models.

- Methods Three different ALS data sets were taken from the same Eucalyptus plantation on the same day, each differing from the others in terms of flight altitude, laser power, and pulse frequency rate. Two sets of best predictive models were obtained for each data set based on two approaches: a basic approach using noncollinear metrics and an exhaustive search, and a second approach that added a pairwise KolmogorovSmirnov test to select stable metrics.

- Results Height metrics proved more stable, especially higher percentiles ( $>50 \%$ ) and the mode. Models developed with stable metrics had similar performance compared to the basic approach.

- Conclusion Percentiles higher than $50 \%$ and the mode proved stable for that 6-year-old Eucalyptus plantation with a very homogeneous vertical structure. Further research widening the scope in terms of age and heterogeneity of vertical profiles is needed.

\author{
C. A. Alvares • O. C. Campoe \\ Forestry Science and Research Institute-IPEF, \\ 13415-000 Piracicaba, SP, Brazil \\ C. A. Alvares \\ e-mail: clayton@ipef.br \\ O. C. Campoe \\ e-mail: otavio@ipef.br
}

J. L. Stape
Department of Forestry and Environmental Resources, North
Carolina State University, Raleigh, NC 27695, USA
e-mail: stape@ncsfnc.cfr.ncsu.edu 
Keywords Light detection and ranging $\cdot$ LiDAR $\cdot$ Vegetation mapping $\cdot$ Monitoring $\cdot$ Forest measurement

\section{Introduction}

A goal of remote sensing in forestry is the reliable prediction of key vegetation parameters from data gathered using aircraft and satellite-borne instruments. In recent years, airborne laser scanning (ALS), otherwise referred to as light detection and ranging (LiDAR), has emerged as the leading technology for the construction of high resolution three-dimensional maps (Lim et al. 2008; Montaghi et al. 2013).

The technology works by sending out short pulses of laser light downward from an airborne platform (airplane). Part of the light is reflected back to the platform from features on the ground, with the return time giving the distance from the platform to the feature. At the moment each laser pulse is sent out, the position of the platform, obtained from an on-board GPS unit, is logged together with the direction of propagation of the light pulse from a highly sensitive inertial measurement unit attached to the beam steering optics (Baltsavias 1999a, b). In practice, a single outgoing pulse may generate multiple returns. This arises when there are partially transmitting strata of objects, such as the leaves and branches of trees, above the ground. Reflected pulses will come from these strata as well as from the light which reaches and is reflected from the ground. The raw vertical heights of the points in the $3 \mathrm{D}$ cloud are relative to a geo-fixed level. These heights can be normalized by subtracting the altitude of the ground (available from the pulses reflected at the ground). This post-processing of the ALS data yields a ground-referenced cloud showing the positions, heights, and shapes of partially transmitting objects, such as trees. It is these unique capabilities which have made ALS a useful remote sensing technology for forestry monitoring and a potential source of vegetation structure information.

Since the number of points in an ALS cloud is very high, for the evaluation of forest, it is usual to reduce the point cloud over each plot to a set of metrics. These metrics are statistics that describe the distribution of the ALS returns. Each return is basically characterized by a ground position, a height above the ground, and an intensity. The point height records for a plot generate a discrete distribution of number of returns for different heights above the ground. The characterization of this distribution is achieved through height metrics of location (for example, mean and percentile boundaries), height metrics of dispersion and form (for example, standard deviation and skewness) (Dalponte et al. 2009), and density metrics (for example, the fraction of points in the distribution with heights greater than the mean) (Næsset 2009).

The distribution of return intensities, relative to the intensity of the incident pulse, for a plot can also be extracted from an ALS data set and summarized by metrics analogous to those used to describe the height distribution. However, many factors influence the intensity of a return, making it hard to obtain reproducible distributions of return intensities (Bater et al. 2011). Therefore, intensity metrics were not computed or analyzed in this study.

The content of an ALS data set is as much influenced by the laser source, the sensor parameters, and the flight settings as by the features present on the ground. Considering a given location on the ground, the number of pulses incident on unit area perpendicular to the vertical (the pulse density) depends on the height and speed of the airborne platform over the ground, the pulse repetition frequency, the angles defining the path of the incident pulses, and the speeds at which the laser scanning optics change these angles. All of these factors are mathematically defined, so that the incident pulse density can be calculated (Baltsavias 1999a).

Forests are considered semipermeable for the laser beam. Trees are spatially complex objects capable of generating many returns from each pulse, thus predicting which of these many returns will be logged by an ALS sensor that is extremely challenging and dependent on many factors. One return point will be recorded when the energy reflected back to the sensor has power enough to trigger the detection algorithm (Chasmer et al. 2006). In order to receive a useful signal from higher-flying heights, both the transmitted power and the dimensions of the receiver optics need to be increased, and the beam divergence decreased (Baltsavias 1999a). It is important to mention that the capacity to register a return point is not only reliant solely upon the ALS equipment but also reliant upon the object. Object reflectivity thus has a fundamental role concerning the quality of the signal.

Differences in point cloud data and in point cloud properties (e.g., point density, pulse density, overlap areas) collected at different times are extremely common, if not inevitable, and these differences must be considered carefully. The most frequent causes of these differences include the use of different instruments and modifications of the flight settings (Bater et al. 2011). A straightforward approach in reducing these impacts is to adjust the model parameters using new field data after a particular ALS survey. Keeping these flight settings constant or collecting a new set of field data will substantially impact upon the cost of surveys (Næsset 2009).

In this study, we examined data sets corresponding to consecutive scans of the same Eucalyptus plantation carried out on the same day using different settings for the equipment and flight. Our aim was to identify stable metrics that could serve as independent variables in stand volume modeling, looking forward to assist Eucalyptus plantation managers by developing transferable models.

The idea of model transferability is to use previously estimated model parameters from a different area for model estimation (Ben-Akiva and Bolduc 1987). Although the predictive ability of a model can be robust within the area for which 
the models have been fitted, their transferability into novel areas is a critical issue (Rödder and Lötters 2010). The identification of stable metrics is essential to develop models which may be transferred to novel areas.

\section{Material and methods}

The study area is located at the geographical coordinates $\left(22^{\circ}\right.$ $58^{\prime} 04^{\prime \prime} \mathrm{S}, 48^{\circ} 43^{\prime} 40^{\prime \prime} \mathrm{W}$ ), which lies within the municipality of Itatinga in the state of São Paulo, Brazil (Fig. 1). It comprises a plantation of 7-year-old Eucalyptus grandis, covering an area of 198.9 ha. The tree spacing was $3.75 \mathrm{~m} \times 1.60 \mathrm{~m}$, resulting in $6 \mathrm{~m}^{-2}$ per tree. The terrain displays gentle undulations (height difference between the highest and lowest points $=57 \mathrm{~m}$ ), with an average elevation of $750 \mathrm{~m}$ above sea level (Campoe et al. 2012). The mean annual rainfall was $1391 \mathrm{~mm}$, with $75 \%$ concentrated from October to March. Mean annual temperature was $19.2{ }^{\circ} \mathrm{C}$, ranging from $13.3^{\circ} \mathrm{C}$ in the coldest months (June to August) to $27.2{ }^{\circ} \mathrm{C}$ in the warmest months (December to February) (Alvares et al. 2013).

The study site was formed of evenly planted trees from superior genetic material; the trees were of the same age and the mortality rate was low. These forest plantation conditions suggest a linear relationship between stand volume and metrics. To ensure this assumption, we explored the data looking for the significance of the linear regression models between ALS metrics and stand volume.

The field data were acquired in July 2009. We used a previous established 12 rectangular plots based on 84 stems in the central blocks and then added more 11 plots of 144 stems to extend the field survey to the entire area (Fig. 1). The plot area was determined by field tape measurements resulting in a range from 500 to $900 \mathrm{~m}^{2}$. To avoid losing the plot during the clear-cut, the limits of each plot were marked by painting the tree's base which corresponded to the boundaries. The height of each tree within a given plot was measured with a hypsometer Haglof HEC-R for height and the diameter at $1.30 \mathrm{~m}$ above the ground with a steel diameter tape. The timber volume for the tree was then estimated from its height and diameter using a formula developed by DURATEX S.A. (forest manager) for $E$. grandis established in this region. The stand volume was standardized in cubic meters per hectare. Uniform clear-cutting of the entire plantation occurred a few days after the tree height, and diameter measurements were completed. The geographical positions of the vertices of each plot could then be determined by differential GPS, with no risk of crown interference. At each vertex, GPS signals were logged using a roving receiver with an external antenna (Trimble Pro XR), and these records were post-processed with correction data retrieved from the fixed base station in Presidente Prudente (station number $93900,22^{\circ} 07^{\prime} 09.9679^{\prime \prime} \mathrm{S}, 51^{\circ} 24^{\prime}$ $28.9700^{\prime \prime} \mathrm{W}$ and altitude of $435.40 \mathrm{~m}$ ) operated by the Brazilian Institute of Geography and Statistics (IBGE) to give final plot corner positions. Basic statistics of the diameter, height, and stand volume over the 23 rectangular plots are presented in Table 1. The differences between plots are related to the topography gradient $(713-767 \mathrm{~m})$, which directly reflects in water disposable, and also to the soil characteristics of the area as detailed by Campoe et al. (2012).

Three ALS data sets (DS) were acquired on April 2009, with a Leica ALS50-II carried on an EMB 810 C SENECA II
Fig. 1 The study lies within coordinates $(731500,7457500)$ and $(734000,7459000)$ SAD69, Zone 23S, in the municipality of Itatinga, São Paulo, Brazil. The rectangular plots were randomly spread out over the study area

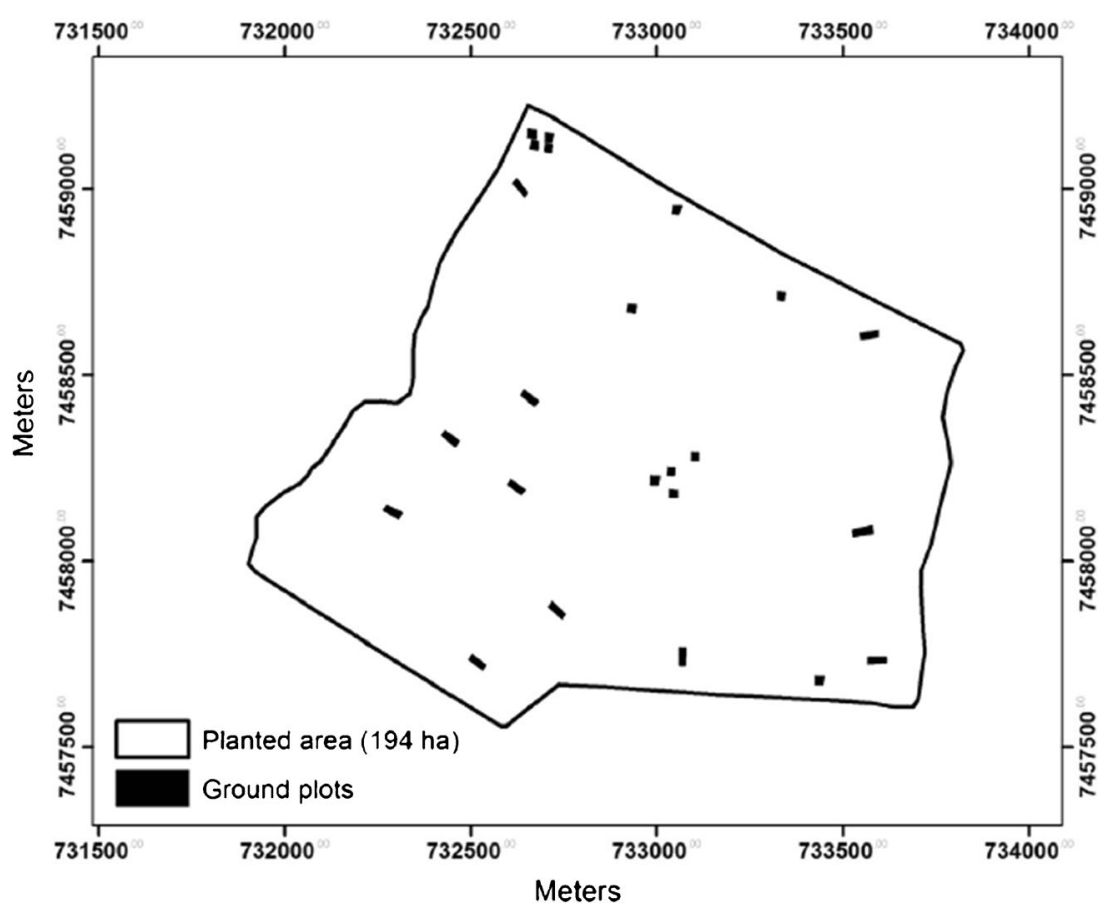

害INRA פ̂spriager 
Table 1 Location statistics of the diameter $(\mathrm{cm})$, height $(\mathrm{m})$, and stand volume $\left(\mathrm{m}^{3} \mathrm{ha}^{-1}\right)$ over 23 plots

\begin{tabular}{lllllll}
\hline & Minimum & $\begin{array}{l}\text { First } \\
\text { quartile }\end{array}$ & Median & Mean & $\begin{array}{l}\text { Third } \\
\text { quartile }\end{array}$ & Maximum \\
\hline Diameter & 41.3 & 44.3 & 47.2 & 47.0 & 49.2 & 52.1 \\
Height & 21.5 & 23.7 & 24.7 & 24.6 & 25.5 & 27.5 \\
Volume & 278.0 & 354.2 & 402.4 & 410.6 & 471.9 & 536.6 \\
\hline
\end{tabular}

aircraft with slightly different settings (Table 2). Each data set contains the results from a series of parallel sweeps over the study area, and the following parameters were the same for every flight: flight speed $\left(140 \mathrm{~km} \mathrm{~h}^{-1}\right)$, half angle $\left( \pm 7.5^{\circ}\right)$, and scan rate $(74 \mathrm{~Hz})$. The lateral displacement of successive sweeps over the study area was set to give $30 \%$ overlap.

The altitude of the terrain beneath each point was subtracted from the altitude of the point. The digital elevation model of each data set required for this normalization was obtained based on the Kraus and Pfeifer filter (Kraus and Pfeifer 2001) to a horizontal resolution of $1 \mathrm{~m}$. The vertical precision between laser scanning surveys expressed by the median of absolute deviation to the median (sigmaMAD as defined by Zlinszky et al. 2013) was in the upper case equal to $0.063 \mathrm{~m}$.

The point cloud regarded to each georeferenced field plot was subsequently normalized by the DTM and its respective metrics calculated. The corresponding metrics extracted from plot level were also calculated for a grid consisting of 20-m square cells, each encompassing a single data set. The $20-\mathrm{m}$ cells were used to approximate the small side of the rectangular field plots.

All metrics were based on the normalized point clouds. Height metrics (HM) calculated from the first and all returns data included the following: mean, quadratic mean, cubic mean, and mode (measures of central tendency); percentiles of $10,25,50,75,90,95$, and 99; and standard deviation, coefficient of variation, skewness, and kurtosis (measures of dispersion) (Magnussen and Boudewyn 1998; Næsset 2009). These 15 height metrics were calculated from the height values of all the points above $1 \mathrm{~m}$ in each cloud and from the heights of the subset of points corresponding to first returns, giving a total of 30 height metrics for each plot and

Table 2 Settings between the three data sets (DS)

\begin{tabular}{llll}
\hline & DS1 & DS2 & DS3 \\
\hline Flight height (m) & 2485 & 1658 & 900 \\
PRF (Hz) & 100.7 & 137.3 & 109.7 \\
Laser power (\%) & 77 & 54 & 28 \\
Number of strips & 4 & 10 & 22 \\
Point density average & 2.8 & 5.6 & 8.7 \\
\hline
\end{tabular}

data set. The density metrics (DM) are ratios (expressed as percentages) of a relation between the total number of points and the numbers of points satisfying the following heightvalue criteria: greater than $2 \mathrm{~m}$, greater than the mean for the plot, and greater than the mode for the plot (Goodwin et al. 2006; Riaño et al. 2004). We generated a total of six density metrics for each plot and data set. The complete list of metrics can be found in Appendix.

The best model for estimating stand volume based on the metrics calculated from all 23 plots was determined for each particular data set. The statistical sequence to model creation was adapted from Stephens et al. (2012). Firstly, we examined the LiDAR metrics to identify highly correlated metrics, through the direct interpretation of the correlation matrix and its classification into several colinearity groups. All metrics with a correlation greater than $90 \%$ were grouped together. Then, to ensure that at most, one variable from each group was included as candidate metric when fitting multiple regression models; the highly correlated metric to stand volume was chosen to represent each group. All of these metrics were then used to perform a regression analysis on the stand volume. Every possible combination was investigated via limiting the regression size up to three independent variables. The best regressions were then selected through the Akaike Information Criteria (AIC).

After determination of the best models considering all metrics for each data set, we started to study the stability of the metrics amongst the data sets. The similarity between a set of metrics at plot level does not guarantee stability for the entire study area, even when the plots are an asymptotically unbiased sample of the population. The ALS data sets, though, covers the entire area returning a dense cloud point sample from the population, capturing much more details and resulting in differences not captured at the plot level. Therefore, the interest on investigating the stability amongst data sets via a comparison of the metrics extracted from 20 -m cell grids.

A paired Kolmogorov-Smirnov test (KS) determined which metrics had nonsignificant differences in the distribution between data sets. Nonsignificant differences between the possible paired comparisons identified the stable metrics. The same method used to group and select variables described to all metrics was applied to the model creation of the stable metrics. Then, the best model for estimating stand volume was fitted considering the candidate stable metrics.

The best models obtained for each data set based on nondiscriminated metrics and the best models using the most stable metrics were then compared and evaluated according to the AIC; the relative root mean square error (rRMSE) was also supplied.

To ensure the transferability capacity of stable metrics, we applied the model developed to one particular data set to estimate the volume of the other two data sets. Then, the KS 


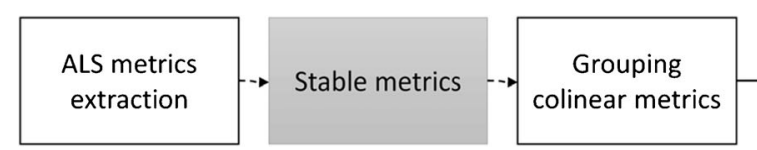

Fig. 2 Stages of the analysis to study the stability of ALS metrics produced for three different ALS data sets that were taken over the same Eucalyptus plantation on the same day and that had only three technical flight parameters altered: elevation, laser power, and pulse

provided a statistical test of equivalence between pairs of 20 $\mathrm{m}$ cell size grids (e.g., the volume map estimated to data set 1 by model 1 against the volume map estimated to data set 2 by model 1 and the volume map estimated to data set 1 by model 1 against volume map estimated to data set 3 by model 1). The same comparison was performed to model 2 while estimating volume from data sets 1 and 3 and model 3 while estimating data sets 1 and 2 . The complete analyses are illustrated in the flowchart presented in Fig. 2.

\section{Results}

The noncollinear groups of metrics varied widely between the data sets. Metrics derived from DS1 to DS2 were grouped into seven different noncollinear groups, while DS3 into nine different groups. Even in the number of noncollinear groups were the same, the group members showed some interesting differences however. Whereas HSD, HCV, HSDf, and HCVf (see Appendix for more information about the metrics) metrics formed a separate group in DS1, they were grouped with the other height metrics for DS2. The metric HP10f was also included in an isolated group for DS2.

When analyzing the groups formed for DS3, the metric HP25 went to an isolated group, HP10f remained isolated as in DS2, and HSD, HCV, HSDf, and HCVf metrics formed a

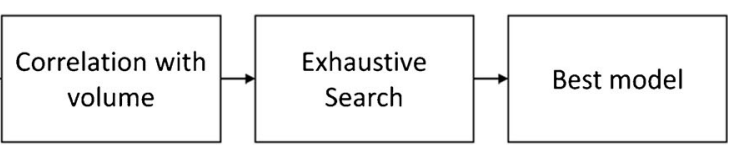

frequency rate. Two sets of best predictive models were obtained for each data set based on two approaches: a basic approach using noncollinear metrics and an exhaustive search, and a second approach that added a pairwise Kolmogorov-Smirnov test to select stable metrics

separate group as in DS1. For all of them, the density metrics were similarly grouped: PARAMO and PFRAMO together, PARA2 and PFRA2 together, and another group consisting of PARAM and PFRAM (Table 3).

Similar to group formation, the metrics which represented each group also differed between the data sets. The metrics representing the groups formed for DS1 were HP50f, HSD, HK, PARA2, PARAM, PARAMO, and HP10. For DS2, the metrics were HP25f, HS, PARA2, PARAM, PARAMO, HP10, and HP10f. Finally, for DS3, the representative metrics were HP25f, HSD, HS, PARA2, PARAM, PARAMO, HP10, HP10f, and HP25.

The KS test provided a statistical test of equivalence of one metric at a time between pairs of grids. From $30 \mathrm{HM}$ to $12 \mathrm{DM}$ including all and first returns, $10 \mathrm{HM}$ and $1 \mathrm{DM}$ displayed nonsignificant differences of cumulative probability distribution between grid cells at a confidence level of $95 \%$ (Table 4 ).

If considering only the stable metrics established by a KS test between DS, the noncollinear groups formed and the representative metrics of each group are the same. The 11 stable metrics between data sets were split into two groups. One containing the height metrics (HMO, HP50, HP75, HP90, HP95, HP99, HMOf, HP90f, HP95f, and HP99f) and the second group containing the density metric PARAMO (Table 5).

We confirmed that the assumption of linearity between the stand volume and the metrics after the linear regressions

Table 3 Groups formed by data set considering the correlation matrix to all extracted metrics

\begin{tabular}{llll}
\hline & DS1 & DS2 & DS3 \\
\hline Group 1 & HM, HMO, HP25, HP50, HP75, & HM, HMO, HP25, HP50, HP75, HP90, & HM, HMO, HP25, HP50, HP75, HP90, \\
& HP90, HP95, HP99, HSQ, HC, & HP95, HP99, HSQ, HC, HMf, HMOf, & HP95, HP99, HSQ, HC, HMf, HMOf, \\
& HMf, HMOf, HP10f, HP25f, & HP25f, HP50f, HP75f, HP90f, HP95f, & HP10f, HP25f, HP50f, HP75f, HP90f, \\
& HP50f, HP75f, HP90f, HP95f, & HP99f, HSQf, HCf, HSD, HCV, & HP95f, HP99f, HSQf, HCf \\
Group 2 & HSD9f, HSQf, HCf & HSDf, HCVf & HSD, HCV, HSDf, HCVf \\
Group 3 & HS, HK, HSf, HKf & HS, HK, HSf, HKf & HS, HK, HSf, HKf \\
Group 4 & PARA2, PFRA2 & PARA2, PFRA2 & PARA2, PFRA2 \\
Group 5 & PARAM, PFRAM & PARAM, PFRAM & PARAM, PFRAM \\
Group 6 & PARAMO, PFRAMO & PARAMO, PFRAMO & PARAMO, PFRAMO \\
Group 7 & HP10 & HP10 & HP10 \\
Group 8 & & HP10f & HP25 \\
Group 9 & & & HP10f \\
\hline
\end{tabular}

In bold, the highest correlated metrics to volume representing each group. The metrics are identified in Appendix, and the data sets in Table 2 
Table $4 \quad p$ value for the nonsignificant metrics by the Kolmogorov-Smirnov test comparing two-by-two distribution metrics extracted for a grid by $20 \times 20 \mathrm{~m}$ superimposed to the study area

\begin{tabular}{llllllllllll}
\hline & HMO & HP50 & HP75 & HP90 & HP95 & HP99 & HMOf & HP90f & HP95f & HP99f & PARAMO \\
\hline DS1 $\times$ DS2 & 0.25 & 0.08 & 0.02 & 0.03 & 0.06 & 0.19 & 0.25 & 0.03 & 0.03 & 0.10 & 0.07 \\
DS2 $\times$ DS3 & 0.74 & 0.81 & 0.56 & 0.32 & 0.20 & 0.30 & 0.55 & 0.31 & 0.24 & 0.24 & 0.33 \\
DS1 $\times$ DS3 & 0.45 & 0.05 & 0.48 & 0.72 & 0.98 & 0.99 & 0.62 & 0.59 & 0.93 & 0.99 & 0.01 \\
\hline
\end{tabular}

The metrics are identified in Appendix, and the data sets (DS) in Table 2

between ALS metrics and stand volume resulted in statistically significant coefficients and models (Fig. 3). The test for linearity was found to be significant, and the nonlinear models (quadratic and cubic) did not increase the explained variance significantly. Thus, the modeling of stand volume was based on multiple linear regression.

While considering all 42 metrics (first and all returns) for both the grouping of metrics and evaluating the best potential model, we did not find any consistency between the best model per data set. The best model for DS1 was built with HP50f and HP10 metrics. For DS2, the best model had one unique metric, the HP25f, and for the DS3, the stand volume was regressed against HP25f and HSD. Between the 11 stable metrics, the metrics that make up the best model were repeatedly the HP50 (Table 6).

When investigating the scenario where the model must be based on one height metric and one density metric, and where all of the 42 metrics are eligible, the best model for DS1 consisted of the metrics HP50f and PARA2. The best model for DS2 consisted of the metrics HP25f and PARAM. The best model for DS3 consisted of the metrics HP25f and PARA2. If narrowing the eligible metrics to solely the stable group, the best model was the same across all data sets and was formed by metrics: HP50 and PARAMO (Table 7).

In the transferability test of the best models based on stable metrics (V EP50), the KS test resulted in a nonsignificant difference between pairs of 20-m grids (Table 8). The grid of volume estimated by model developed and applied to DS1 had no statistical differences when applied to DS2 neither DS3. The same results were obtained to DS2 model applied to DS1 and DS3, and DS3 model applied to DS1 and DS2.

Table 5 Groups formed by data set considering just nonsignificant metrics under KS test

\begin{tabular}{ll}
\hline \multicolumn{1}{c}{ DS1 } \\
\hline $\begin{array}{l}\text { Group 1 } \\
\text { HMO, HP50, HP75, HP90, HP95, HP99, HMOf, HP90f, } \\
\text { HP95f, HP99f }\end{array}$ \\
Group 2 & PARAMO
\end{tabular}

In bold, the highest correlated metrics to volume representing the group. The metrics are identified in Appendix, and the data sets in Table 2

\section{Discussion}

Three data sets obtained for the same area in the same day, but employing different equipment configurations and different flight settings, generated slightly different metrics. These differences appeared both in the correlation between metrics, as well as in the correlation to the dependent variable (stand volume). The implication here is that the best model in estimating stand volume also differs between the data sets.

Thomas et al. (2006) found different models for each ALS data set comparing the coefficient of determination and the root mean square error. The best model to estimate stem volume by their low-density data set $\left(0.035 \mathrm{pts}^{-2}\right)$ used the first returns percentile 50 and the best model to their high-density $\left(4 \mathrm{pts}^{-2}\right.$ ) data set used the all returns percentile 50 . Gonzalez-Ferreiro et al. (2012) have also explored the selection of height metrics for the prediction of stand volumes from different LiDAR data sets. The best models for their $0.5 \mathrm{pts}^{-2}$ data set were used as predictors of the height metrics: standard deviation, skewness, and the percentile 5 . In contrast, the stand volume model for the $8 \mathrm{pts}^{-2}$ data set did not include the standard deviation.

Application of the KS test to compare cell metric values between pairs of data sets showed statistically significant differences between the data sets for most metrics. The exceptions were the high height percentiles, the mode of the heights, and the proportion of all returns above mode, which displayed statistical similarity between all the data sets.

High percentiles are less sensitive to outliers than metrics such as maximum height, which can be skewed by a small portion of the area with abnormally high material or even by returns from flying birds (Kane et al. 2010). On the other hand, lower percentiles might be influenced by the signal attenuation consequence of the higher altitude. The overall control on the magnitude of the return will be the strength of the pulse energy emitted. Sensor and flight settings directly control the energy and size of the emitted pulse, and the recorded intensity is directly related to the magnitude of the returned peak of the recorded backscatter (Hopkinson 2007). As was shown by Chasmer et al. (2006), who studied the effect of pulse energy on canopy penetration and found that pulses with 

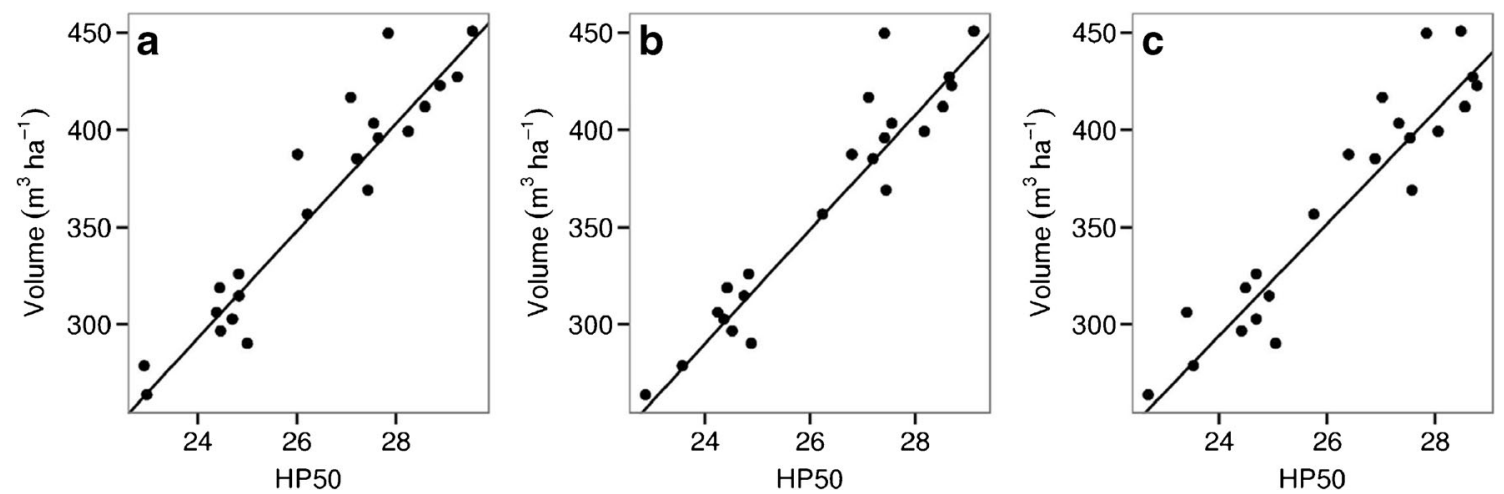

Fig. 3 Linear regression observed between the HP50 metric and the stand volume for data sets 1 (a), 2 (b), and 3 (c). The equations used to plot the line are identified in Table 8

higher energy penetrate further into the canopy, the acquisition settings of modern small footprint systems do influence laser metrics.

Flight settings as platform altitude and scan angle (measured off nadir) are related to pulse return intensity as greater distances from target to sensor will exponentially reduce the intensity of returned energy given the assumption of isotropic scattering (Newton's Inverse Distance Law). Discrete recording LiDAR systems have been designed to detect returns above a preset energy threshold, below which returns cannot be distinguished from random noise. As a consequence, a reduction in the detected intensity of laser light will result in the detection of fewer laser returns. This will have a stronger effect on soft targets, where the laser light is scattered from a range of small surfaces at different angles and the intensity of reflected light from each distinct surface is characteristically lower (Goodwin et al. 2006).

Hopkinson (2007) also alerts that tall vegetation attenuates more of the emitted pulse energy, effectively trapping it in the canopy. The intensity of split returns from multiple small surfaces along the pulse path length will tend to be lower than

Table 6 The best model to estimate stand volume based on ALS metrics

\begin{tabular}{llll}
\hline DS/metrics & Best model & AIC & rRMSE \\
\hline DS1/all metrics & $V=$ HP50f+HP10 & 224.7 & 7.51 \\
DS2/all metrics & $V=$ HP25f & 220.4 & 7.14 \\
DS3/all metrics & $V=$ HP25f + HSD & 223.4 & 7.31 \\
DS1/stable metrics & $V=$ HP50 & 224.4 & 7.80 \\
DS2/stable metrics & $V=$ HP50 & 223.8 & 7.69 \\
DS3/stable metrics & $V=$ HP50 & 225.4 & 7.97 \\
\hline
\end{tabular}

The metrics are identified in Appendix, and the data sets in Table 2 $r M S E$ relative root mean square error that of single returns from a single larger backscatter surface. In general, reduced pulse energy concentration appears to reduce pulse penetration through foliage to ground level due to increased pulse attenuation in the canopy causing a slight upward bias in last return ground elevations. The important exception to this observation is in high pulse repetition frequency (PRF) data, where reduced pulse power concentration effects are overshadowed by increased noise in the data (short vegetation) and increased sample point density (tall vegetation).

The sensitivity of density metrics is reflected in the absence of this group of metrics among the best models selected for each data set (Table 6). The density metric incorporated a measure of the number of times the low part of the vegetation was sensed and a measure of canopy height differences. As the canopy opens up, the number of pulses direct to ground and the number of pulses where ground is sensed increases (Nelson et al. 1988). The reference height may be fixed or otherwise defined by a function of other height metrics (e.g., mean, mode) (Næsset 2002; McGaughey 2013). The density metric computations depends itself of the height metrics properties. In case when a fixed reference height is selected, it is usually close to the lower percentiles, which were considered unstable between the flights. The unique density metric considered stable was PARAMO that considers the mode as the

Table 7 The best model with one height metrics and one density metric to estimate stand volume based on stable ALS metrics

\begin{tabular}{llll}
\hline DS & Best model & AIC & rRMSE \\
\hline 1 & $V=$ HP50+PARAMO & 226.1 & 7.74 \\
2 & $V=$ HP50+PARAMO & 225.3 & 7.61 \\
3 & $V=$ HP50+PARAMO & 227.2 & 7.93 \\
\hline
\end{tabular}

The metrics are identified in Appendix, and the data sets in Table 2 $D S$ data sets, $A I C$ Akaike Information Criteria, $r M S E$ relative root mean square error 
Table 8 Kolmogorov-Smirnov test comparing the 20-m cell size grid of stand volume estimated by model ( $V=\mathrm{HP} 50)$ developed from different a data set and the model applied to its respective data set

\begin{tabular}{lccc}
\hline & DS1 & DS2 & DS3 \\
\hline $\mathrm{DS} 1 / V=-369.31 *+27.59 *$ HP50 & & 0.08 & 0.05 \\
$\mathrm{DS} 2 / V=-415.39^{*}+29.39 *$ HP50 & 0.08 & & 0.81 \\
$\mathrm{DS} 3 / V=-396.85^{*}+28.79 *$ HP50 & 0.05 & 0.81 & \\
\hline
\end{tabular}

The metrics are identified in Appendix, and the data sets in Table 2

${ }^{*} p$ value $<0.001$

reference height. In our study, mode was also a stable height metric.

These high sensitivities also due to the fact that as altitude increases footprints also increase, returns densities decrease, and irradiance per unit area decreases. Therefore, as the flight altitude varies, full waves may be trigged and decomposed into returns differently from flight to flight. Nonetheless, Bater et al. (2011), when testing the reproducibility of ALS metrics with multiple scans over the same location taken on the same day, found that the majority of the height metrics was not significantly different. However, the differences for the density metrics were highly significant and arose from large differences in point density between the four flight lines. They argued that the observed differences in point density found during their campaign might have been caused by unintentional variations in the orientation and altitude of the platform.

Lim et al. (2008) suggested that "changes in point density (due to changes in scan angle and altitude) only affect laser canopy height and density metrics that are characterized by the small percentage of returns from the very top (e.g., maximum height; proportion of returns bellow percentile $\left.10^{\text {th }}\right)$ and base of the canopy (minimum height; percentile of return above percentile $90^{\text {th }}$ ) (i.e., those metrics that characterize the tail ends of the distributions of laser canopy heights)."

Once the candidate metrics as explanatory variable for stand volume had been limited to metrics that were considered stable, similar models were determined for the data sets, both in respect to performance and to selected metrics. Even equipment configurations and flight parameters that resulted in a higher return density did not show any significant differences. Thomas et al. (2006) also found that an increase in point density does not necessarily improve the prediction accuracy of mean dominant height, basal area, crown closure, and average aboveground biomass.

Even when restricting the candidate metrics to just the stable group, the model performances are still very close (Tables 6 and 7). One particular height metric and one particular density metric were imposed onto the model in order to simulate the biological condition, concerning the site quality and the stand density respectively. The models even in this scenario maintained a similar level of performance to models based upon all of the metrics. This biological condition derived from initial studies, which suspected that the laser response to a forest canopy was solely not only a function of tree height but also a function of canopy closure and density (Nelson et al. 1984). The relationship between the stand variables and the laser data was proved dependent not only on the laser canopy height distribution but also on the canopy cover. Canopy density is of particular importance to explain the variability in stand density (Næsset 2004). Lefsky et al. (2002) concluded that simple models can reproduce emergent community-level relationships, starting with the known allometric properties of plants and incorporating competition for light and space. In a further study, Lefsky et al. (2005) goes beyond discussing that maximum height whose regression from the LiDAR canopy structure indices had already explained $89 \%$ of variance. Therefore, the $15 \%$ of variance in the normalized residuals could represent no more than $2.65 \%$ of the overall relationship between canopy structure, environment, and the stand structure variable.

The use of height metrics and density metrics is very common but not a consensus. Mean height as height metric and a crown cover as density metric were used for predicting stand volume by Holmgren et al. (2003). Usually after a height metrics was included in the regression model, density metrics provided a limited significant improvement in model performance (Nelson et al. 1988).

Foody et al. (2003) explored the problems associated with attempts to transfer predictive models of vegetation toward combined uses with remote sensing data and demonstrated that there exist many complicating factors. The inability to transfer these data relationships satisfactorily seriously limits the contribution that remote sensing can make in environmental applications. Our study has shown that some metrics do nevertheless have the desirable property of returning similar values across different data sets. Once variance in airborne laser scanner sensors and flight settings is considered, the scope for metric values to change in response to properties unrelated to the vegetation being surveyed increases (Magnusson, Fransson, and Holmgren 2007) and so also does the difficulties in the search for transferable models. A straightforward approach toward consistently producing good models is to always use field data when resetting model parameters after a LiDAR data survey. However, a more lowcost method over the long-term would be the discovery and development of transferable models. This would require stable metrics, such as those which show the greatest similarity between different data sets and different methods of remote data gathering.

Even the ALS sensors have evolved over the last decades, the vegetation quantification remains holding in the metrics correlation to biophysical parameters. The use of stable metrics can improve the compatibility between surveys, making 
the results comparable and reducing noise between data sets allowing a historical series studies.

This study investigated candidate metrics to form transferable models based on ALS-derived metrics derived from multiple surveys of data collected at the same site on a particular date. We have investigated the stability of metrics at both the plot and grid levels and have demonstrated how the selection of stable metrics can contribute to generate reliable models between different data sets. According to our results, the height metrics provide the greatest stability when used in the models, specifically the higher percentiles $(>50 \%)$ and the statistic mode.
Acknowledgments This paper is part of the research program developed by GET-LiDAR. The EUCFLUX project, Duratex S.A., the Forestry Science and Research Institute (IPEF), North Carolina State University (NCSU), and Virginia Polytechnic Institute and State University (VT) are acknowledged for their support through the provision of the laser and field data sets. We thank the anonymous reviewers and the editor for their insightful suggestions.

Funding The airborne data acquisition was funded by the EUCFLUX project, the Forestry Science and Research Institute (IPEF), North Carolina State University (NCSU), and Virginia Polytechnic Institute and State University (VT). The field data collection was funded by DURATEX S. A. The Coordenação de Aperfeiçoamento de Pessoal de Nível Superior, CAPES, supported with a student grant, and the ForEAdapt (FP7-PEOPLE-2009-IRSES) supported with a visit to UEF/Finland during data processing.

\section{Appendix}

Table 9 List of computed metrics of height and cover

\begin{tabular}{|c|c|c|c|}
\hline Metric & Description/statistic & $\begin{array}{l}\text { Symbol for all } \\
\text { returns (HM/AR) }\end{array}$ & $\begin{array}{l}\text { Symbol for first } \\
\text { returns }(\mathrm{HM} / \mathrm{FR})\end{array}$ \\
\hline \multicolumn{4}{|l|}{ Height Metrics (HM) } \\
\hline \multicolumn{4}{|l|}{ Location "center" } \\
\hline Mean & $\bar{x}=\frac{1}{n} \sum x_{i}$ & HME & HMEf \\
\hline Quadratic mean & $\sqrt{\frac{1}{n} \sum x_{i}^{2}}$ & HSQ & HSQf \\
\hline Cubic mean & $\sqrt[3]{\frac{1}{n} \sum x_{i}^{3}}$ & $\mathrm{HC}$ & $\mathrm{HCf}$ \\
\hline Mode & Mo $=$ Most frequent value in data set & $\mathrm{HMO}$ & HMOf \\
\hline \multicolumn{4}{|l|}{ Location "percentile" } \\
\hline Percentile & $\begin{array}{l}\text { Height separating lowest } y \% \text { of data } \\
\text { from remainder }\end{array}$ & HPy & HPyf \\
\hline \multicolumn{4}{|l|}{ Dispersion } \\
\hline Standard deviation & $\sqrt{\frac{1}{n-1} \sum\left(x_{i}-\bar{x}\right)^{2}}$ & HSD & HSDf \\
\hline Coefficient of variation & $\frac{\sqrt{\frac{1}{n-1} \sum\left(x_{i}-\bar{x}\right)^{2}}}{\bar{x}}$ & $\mathrm{HCV}$ & $\mathrm{HCVf}$ \\
\hline Skewness & $\frac{\frac{1}{n} \sum\left(x_{i}-\bar{x}\right)^{3}}{\left(\frac{1}{n} \sum\left(x_{i}-\bar{x}\right)^{2}\right)^{3 / 2}}$ & HS & HSf \\
\hline Kurtosis & $\frac{\frac{1}{n} \sum\left(x_{i}-\bar{x}\right)^{4}}{\left(\frac{1}{n} \sum\left(x_{i}-\bar{x}\right)^{2}\right)^{2}}-3$ & HK & HKf \\
\hline \multicolumn{4}{|l|}{ Cover metrics (CM) } \\
\hline \multicolumn{4}{|l|}{ All returns differentiated by height $(\mathrm{CM} / \mathrm{AR})$} \\
\hline Percentage of all returns above a specified height & $\frac{N\left(>h_{j}\right)}{N} \times 100$ & PARA2 & \\
\hline Percentage of all returns above the mean height & $\frac{N(>\bar{h})}{N} \times 100$ & PARAM & \\
\hline $\begin{array}{l}\text { Percentage of all returns above the mode height } \\
\text { First returns differentiated by height }(\mathrm{CM} / \mathrm{FR})\end{array}$ & $\frac{N(>M o(h))}{N} \times 100$ & PARAMO & \\
\hline Percentage of first returns above a specified height & \multirow{4}{*}{$\begin{array}{l}\frac{N_{1}\left(>h_{j}\right)}{N} \times 100 \\
\frac{N_{1}(>\bar{h})}{N} \times 100 \\
\frac{N_{1}(>M o(h))}{N} \times 100 \\
\frac{N_{1}\left(>h_{j}\right)}{N_{1}} \times 100\end{array}$} & & PFRA2 \\
\hline Percentage of first returns above the mean height & & & PFRAM \\
\hline Percentage of first returns above the mode height & & & PFRAMO \\
\hline $\begin{array}{l}\text { Percentage of first returns above the specified height } \\
\text { related to first returns }\end{array}$ & & & FR2FR \\
\hline
\end{tabular}




\section{References}

Alvares CA, Stape JL, Sentelhas PC, de Moraes Goncalves JL, Sparovek G (2013) Koppen's climate classification map for Brazil. Meteorol Z 22:711-728

Baltsavias EP (1999a) Airborne laser scanning: basic relations and formulas. ISPRS J Photogramm Remote Sens 54:199-214

Baltsavias EP (1999b) Airborne laser scanning: existing systems and firms and other resources. ISPRS J Photogramm Remote Sens 54: 164-198

Bater CW, Wulder MA, Coops NC, Nelson RF, Hilker T, Naesset E (2011) Stability of sample-based scanning-LiDAR-derived vegetation metrics for forest monitoring. [article]. IEEE Trans Geosci Remote Sens 49:2385-2392

Ben-Akiva, M, Bolduc, D (1987) Approaches to model transferability and updating: the combined transfer estimator (No. 1139)

Campoe OC, Stape JL, Laclau J-P, Marsden C, Nouvellon Y (2012) Stand-level patterns of carbon fluxes and partitioning in a Eucalyptus grandis plantation across a gradient of productivity, in São Paulo State, Brazil. Tree Physiol 32:696-706

Chasmer L, Hopkinson C, Treitz P (2006) Investigating laser pulse penetration through a conifer canopy by integrating airborne and terrestrial lidar. Can J Remote Sens 32:116-125

Dalponte M, Coops NC, Bruzzone L, Gianelle D (2009) Analysis on the use of multiple returns LiDAR data for the estimation of tree stems volume. IEEE J Sel Top Appl EarthObs Remote Sens 2:310-318

Foody GM, Boyd DS, Cutler MEJ (2003) Predictive relations of tropical forest biomass from Landsat TM data and their transferability between regions. Remote Sens Environ 85:463-474

Gonzalez-Ferreiro E, Dieguez-Aranda U, Miranda D (2012) Estimation of stand variables in Pinusradiata D. Don plantations using different LiDAR pulse densities. Forestry 85:281-292

Goodwin NR, Coops NC, Culvenor DS (2006) Assessment of forest structure with airborne LiDAR and the effects of platform altitude. Remote Sens Environ 103:140-152

Hopkinson C (2007) The influence of flying altitude, beam divergence, and pulse repetition frequency on laser pulse return intensity and canopy frequency distribution. Can J Remote Sens 33:312-324

Holmgren J, Nilsson M, Olsson H (2003) Estimation of tree height and stem volume on plots using airborne laser scanning. For Sci 49: 419-428

Kane VR, McGaughey RJ, Bakker JD, Gersonde RF, Lutz JA, Franklin JF (2010) Comparisons between field-and LiDAR-based measures of stand structural complexity. Can J For Res 40:761-773

Kraus K, Pfeifer N (2001) Advanced DTM generation from LIDAR data. Int Arch Photogramm Remote Sens Spat Inf Sci 34:23-30

Lefsky MA, Cohen WB, Harding DJ, Parker GG, Acker SA, Gower ST (2002) Lidar remote sensing of above-ground biomass in three biomes. Glob Ecol Biogeogr 11:393-399
Lefsky MA, Hudak AT, Cohen WB, Acker SA (2005) Geographic variability in lidar predictions of forest stand structure in the Pacific Northwest. Remote Sens Environ 95:532-548

Lim K, Hopkinson C, Treitz P (2008) Examining the effects of sampling point densities on laser canopy height and density metrics. For Chron 84:876-885

Magnussen S, Boudewyn P (1998) Derivations of stand heights from airborne laser scanner data with canopy-based quantile estimators. Can J For Res 28:1016-1031

Magnusson M, Fransson JES, Holmgren J (2007) Effects on estimation accuracy of forest variables using different pulse density of laser data. For Sci 53:619-626

McGaughey, RJ (2013) FUSION/LDV: software for LiDAR Data Analysis and Visualization. USDA/Forest Service

Montaghi A, Corona P, Dalponte M, Gianelle D, Chirici G, Olsson H (2013) Airborne laser scanning of forest resources: an overview of research in Italy as a commentary case study. Int J Appl Earth Obs Geoinformation 23:288-300

Næsset E (2002) Predicting forest stand characteristics with airborne scanning laser using a practical two-stage procedure and field data. Remote Sens Environ 80:88-99

Næsset E (2004) Accuracy of forest inventory using airborne laser scanning: evaluating the first Nordic full-scale operational project. Scand J For Res 19:554-557

Nelson R, Krabill W, Maclean G (1984) Determining forest canopy characteristics using airborne laser data. Remote Sens Environ 15:201212

Nelson R, Krabill W, Tonelli J (1988) Estimating forest biomass and volume using airborne laser data. Remote Sens Environ 24:247-267

Næsset, E (2009) Effects of different sensors, flying altitudes, and pulse repetition frequencies on forest canopy metrics and biophysical stand properties derived from small-footprint airborne laser data. Remote Sens Environ 113(1):148-149

Riaño D, Valladares F, Condés S, Chuvieco E (2004) Estimation of leaf area index and covered ground from airborne laser scanner (Lidar) in two contrasting forests. Agric For Meteorol 124:269-275

Rödder D, Lötters S (2010) Explanative power of variables used in species distribution modelling: an issue of general model transferability or niche shift in the invasive Greenhouse frog (Eleutherodactylusplanirostris). Naturwissenschaften 97:781-796

Stephens PR, Kimberley MO, Beets PN, Paul TSH, Searles N, Bell A et al (2012) Airborne scanning LiDAR in a double sampling forest carbon inventory. Remote Sens Environ 117:348-357

Thomas V, Treitz P, McCaughey JH, Morrison I (2006) Mapping standlevel forest biophysical variables for a mixed wood boreal forest using lidar: an examination of scanning density. Can J For ResRev Can Rech For 36:34-47

Zlinszky A, Ressl C, Timár G, Weber R, Székely B, Briese C, Pfeifer N (2013) A proof of concept: airborne LIDAR-measured ellipsoidal heights of a lake surface correspond to a local geoid model. J Conf Abstr EUG 15:10280 